\title{
Trabajo infantil ambulante en las capitales latinoamericanas
}

\author{
Angela Ma. Pinzón-Rondón, MD, MPH,(1) Leonardo Briceño-Ayala, MD,(1) Juan Carlos Botero, JD, LLM, (2) \\ Patricia Cabrera, MD, (I) María Nelcy Rodríguez, MPH.(I)
}

\section{Pinzón-Rondón AM, Briceño-Ayala L, Botero JC, Cabrera P, Rodríguez MN. \\ Trabajo infantil ambulante en las capitales latinoamericanas. Salud Publica Mex 2006;48:363-372.}

\section{Resumen}

Objetivo. Conocer edad, sexo, desplazamiento, escolaridad, actividad desarrollada, jornada laboral, vivienda en calle y cobertura de seguridad social de un grupo de niños que laboran en las calles de las capitales latinoamericanas. Material y métodos. Estudio de corte transversal. Se aplicó un cuestionario a 972 niños que trabajan en las calles de Bogotá, Ciudad de Guatemala, Ciudad de México, Quito y San Salvador, Resultados. El 63.3\% era de sexo masculino; $39 \%$ provenía de familias desplazadas; $18 \%$ habitaba en la calle; $62 \%$ trabajaba más de 40 horas a la semana; 19\% tenía seguridad social, y $32 \%$ se dedicaba al comercio ambulante. Se encontró que la mayoría de las variables se comportan de forma significativamente diferente para cada ciudad. Conclusión. El trabajo infantil ambulante es una actividad peligrosa, de largas jornadas laborales, con exposición a múltiples factores de riesgo, y se comporta de manera significativamente diferente en cada ciudad estudiada. Esto sugiere que las soluciones al problema deben diseñarse caso por caso.

Palabras clave: trabajo infantil, condiciones de trabajo, jornada laboral, comercio ambulante, América Latina
Pinzón-Rondón AM, Briceño-Ayala L, Botero JC, Cabrera P, Rodríguez MN.

Street child work in Latin American capitals.

Salud Publica Mex 2006;48:363-372.

\section{Abstract}

Objective. To identify the age, sex, mobility, education, work activity, working hours, street dwelling, and social security coverage in a group of children working in the streets in capital cities in Latin America. Material and Methods. Cross sectional study. A questionnaire was applied to 972 children working in the streets of Bogotá, Guatemala City, Mexico City, Quito and San Salvador. Results. A total of $63.3 \%$ subjects were boys; $39 \%$ were children from displaced families; $18 \%$ lived in the streets; $62 \%$ worked more than 40 hours per week; $19 \%$ were covered by the social security system, and $32 \%$ were street vendors. The behavior of variables differed significantly by city. Conclusion. Child labor in the streets is a dangerous activity characterized by long working hours and exposure to risk factors. Child work has different characteristics in each of the cities studied, which suggests that the solution to the problem must be designed on a case by case basis.

Key words: child labor, working conditions, working hours, street labor, Latin America

(I) Facultad de Medicina, Universidad del Rosario. Bogotá, Colombia.

(2) Director de la Oficina Comercial de Colombia en EUA.

Fecha de recibido: 21 de octubre de 2005 - Fecha de aprobado: 16 de junio de 2006 Solicitud de sobretiros: Angela María Pinzón Rondón. Cra. 19, 106-18, Ap 201. Bogotá, Colombia. Correo electrónico: ampinzon@urosario.edu.co 
E trabajo infantil es un fenómeno indicativo de las condiciones económicas, sociales y políticas de una región. Las cifras de trabajo infantil presentadas internacionalmente muestran que existen entre 250 y 352 millones de niños trabajadores en el mundo. ${ }^{1}$ De éstos, $48 \%$ se encuentra en Asia; $32 \%$, en África, y 7\%, en América Latina. Sin embargo, la dimensión real del problema en América Latina se percibe cuando se observan las cifras relativas. En esta región, al igual que en Asia, $20 \%$ de la población infantil trabaja. ${ }^{2}$ El fenómeno está altamente asociado con factores como la pobreza, el desempleo, la falta de escuelas, la distribución inequitativa de los recursos, las discriminaciones de género, raza y clase social, las guerras y conflictos, y las definiciones inadecuadas de la edad adulta. ${ }^{3,4}$

Muchos de los menores que trabajan en los países latinoamericanos lo hacen en las calles. Como ejemplo, algunos estudios muestran que, en Bogotá, 57.3\% de los niños trabajadores se dedica al comercio en las calles. 5,6 En la capital de México, el trabajo infantil ambulante se concentra en los cuatro primeros sectores de la ciudad, y se estima que existen 1500000 niños que trabajan en las calles. ${ }^{7}$ Este es un fenómeno en aumento que, tan solo en Centroamérica, se ha incrementado $140 \%$ durante los últimos 20 años. ${ }^{8}$

El trabajo infantil es un flagelo que ha despertado la preocupación de las Naciones Unidas en Latinoamérica, por lo que los países seleccionados en este estudio forman parte del Programa Internacional para la Eliminación del Trabajo Infantil (IPEC, por sus siglas en inglés) de la Organización Internacional del Trabajo (OIT). Esto demuestra que en los países estudiados no se han desarrollado estrategias que contengan o prevengan el fenómeno de manera eficiente, y que las implementadas van dirigidas al control del trabajo infantil en sectores formales de la economía, en su mayor parte. ${ }^{9}$ El propósito final de este estudio es considerar las variables que permitan entender el trabajo infantil en las calles de algunas ciudades de América Latina y proponer estrategias para su erradicación.

\section{Material y métodos}

Este documento contiene los resultados de un análisis de corte transversal sobre el fenómeno del trabajo infantil ambulante de algunas capitales latinoamericanas con un alto índice de niños en las calles: Bogotá, Ciudad de Guatemala, Ciudad de México, Quito y San Salvador. El problema ha sido documentado desde hace varios años en México, Guatemala y Ecuador, en relación con la salud y las condiciones de vida. ${ }^{10}$ Igualmente ha ocurrido en Colombia ${ }^{5}$ y El Salvador. ${ }^{11}$
Además, se han realizado estudios previos que documentan el problema, pero no comparan sus variables de manera sistemática con otros países. ${ }^{13-17}$

La población objeto del estudio correspondió a los menores de 18 años que se encontraban en la calle desarrollando alguna actividad que produjera ingresos, ya fueran para ellos directamente o para las personas que los acompañaban.

Para la recolección de la muestra se seleccionaron de antemano las calles de la ciudad donde, por consenso de los habitantes, se encontraba el mayor número de niños trabajadores. La entrevista se aplicó a quienes se encontraban laborando los días del fin de semana seleccionado. Las entrevistas se llevaron a cabo entre noviembre de 2003 y junio de 2004.

El cálculo de la muestra se determinó con base en la variable "número de horas de trabajo en calle", tomando como punto de corte 20 horas a la semana, por considerar que quienes trabajan medio tiempo o más están significativamente más expuestos a todos los riesgos laborales.

El tamaño de la muestra se calculó utilizando la formula $n=Z^{2} p(1-p) / d^{2} \times$ DEFF/1-TNR para estudios de corte transversal, donde $n$ es el número de sujetos requeridos, $p$ es la probabilidad esperada de que se presente el evento de interés, DEFF es el efecto del diseño y TNR es la tasa de no respuesta. Teniendo en cuenta las características del estudio, se utilizó un efecto de diseño de 1.8 , un error alrededor del estimativo de $5 \%$, y una tasa de falta de respuesta de $5 \%$. A partir de los resultados de un estudio previo realizado en la ciudad de Bogotá, ${ }^{1}$ se estimó inicialmente una prevalencia de 96.5\% de menores, que por trabajar en las calles, laboraban más de medio tiempo. Este dato se consideró extrapolable a las otras ciudades, ya que las jornadas laborales prolongadas para los menores trabajadores son comunes en El Salvador, ${ }^{13}$ Ecuador, ${ }^{14}$ Guatemala ${ }^{15}$ y México. ${ }^{16}$ De acuerdo con los datos anteriores, se estimó un tamaño de muestra inicial de 98 sujetos, el cual se aproximó a 100. Una vez obtenidos los datos de cada ciudad, se ajustó la muestra a los datos obtenidos y se añadieron entrevistas en las ciudades que así lo requirieron. Con base en dicho cálculo, se recolectaron 481 entrevistas en Bogotá; 121, en Ciudad de Guatemala; 100, en Ciudad de México; 180, en Quito, y 100 , en San Salvador.

Como criterios de inclusión se tomaron en cuenta dos factores: edad menor de 18 años y encontrarse trabajando en las calles escogidas de cada ciudad los días determinados. No se determinó un límite inferior a la edad al considerar que los niños pequeños que acompañaban a los adultos a trabajar en las calles también 
estaban expuestos a los factores de riesgo. Como criterio de exclusión se estableció el hecho de que el niño o su familia no deseara participar en el estudio.

Como instrumento de recolección, se utilizó una entrevista que ya había sido utilizada en un estudio anterior sobre trabajo infantil ${ }^{12}$ a la que se le hicieron algunas modificaciones idiomáticas, sugeridas para cada ciudad. El cuestionario no se generalizó ni se utilizó para controlar la aparición de sesgos no muestrales.

Las personas encargadas de recolectar la información en cada una de las ciudades recibieron un protocolo de investigación, un manual para la aplicación de los cuestionarios y entrenamiento. Estas personas tienen un grado universitario y, en su mayoría, son especialistas en salud pública o en medicina ocupacional.

Las variables empleadas para el análisis fueron país, ciudad, acompañante, número de niños presentes, persona que responde la encuesta, edad, sexo, vivienda en calle, desplazamiento, desplazamiento por violencia, tiempo en la ciudad, estudio, retraso escolar, tiempo que permanece en la calle durante una semana, tiempo que permanece por la noche en la calle durante una semana, actividad que desempeña y seguridad social.

Para la digitación de los datos se utilizó el programa Excel. Cada una de las respuestas de los cuestionarios fue digitada y posteriormente el coordinador del estudio revisó la entrada de los datos. Para constatar la calidad del dato y el flujo de información se verificaron todos los filtros y los rangos de valores posibles en todas las variables de interés.

El análisis estadístico se realizó utilizando el programa STATA 7.0. Dadas las características descriptivas del estudio, se recurrió a medidas descriptivas como frecuencias simples, proporciones y promedios según la escala de medición de la variable. Para evaluar la tendencia de las variables más importantes se usaron tablas de contingencia para datos categóricos. Estas últimas se analizaron de acuerdo con la población general y por ciudad. Para estudiar las posibles asociaciones entre las variables de interés y algunos potenciales factores asociados se utilizó la prueba de ji cuadrada. Finalmente se realizó un análisis de correspondencias entre las diferentes ciudades con el fin de agrupar variables que permitieran establecer un perfil del problema.

La participación en el estudio fue de carácter voluntario y anónimo. El estudio siguió las normas referentes a investigación de cada país, y se guía por la Declaración de Helsinki y sus modificaciones en lo referente a ética.

\section{Resultados}

De 982 entrevistas realizadas, fue necesario eliminar 10 que no cumplían con los criterios de inclusión, lo que dio un total de 972 cuestionarios. De éstos, 478 pertenecían a Bogotá (49.2\%); 120 a la Ciudad de Guatemala (12.3\%); 97 a la de México (10\%); 177 a Quito $(18.2 \%)$, y 100 a San Salvador (10.3\%).

\section{Generalidades}

De los niños entrevistados, $46.7 \%$ se encontraba $\sin$ la compañía de un adulto y $57.2 \%$ se encontraba en grupos de dos niños por lo menos. En $66.9 \%$ de los casos fue el niño quien respondió el cuestionario, mientras que en $25.1 \%$ fue su acompañante adulto; en $8 \%$ de los casos, fue contestado entre el niño y su acompañante. De los niños incluidos en la encuesta, eran varones $63.2 \% ; 2.3 \%$ era menor de un año; $16.2 \%$ tenía entre uno y cinco años; $64.2 \%$, entre 6 y 14 años, y $17.4 \%$, entre 15 y 17 años de edad. $72.2 \%$ no tenía seguridad social; $18.9 \%$ sí la tenía y $8.9 \%$ desconocía si la tenía o no (cuadro I).

\section{Permanencia en calle}

De los niños entrevistados, $17.7 \%$ se encontraba viviendo en calle; $9.7 \%$ reportó que trabajaba en la calle menos de 20 horas a la semana; $28.2 \%$, entre 20 y 39 horas; $62.1 \%$, más de 40 horas a la semana, y $29.3 \%$ realizaba trabajo nocturno. En relación con este último aspecto, $3.3 \%$ trabajaba entre 1 y 9 horas en la noche, y $26 \%, 10$ horas o más (cuadro II).

\section{Desplazamiento}

Del total de los niños, $39 \%$ era hijo de familia desplazada y el $26.7 \%$ de familia desplazada por la violencia. De los desplazados, $43 \%$ llevaba menos de un año en las ciudades, y tenía menor probabilidad de contar con seguridad social que el resto de la población estudiada $(p=0.018)$ (cuadro III).

\section{Características de actividad}

El 31.6\% de los niños se dedicaban al comercio ambulante; $14.9 \%$, a pedir limosna; $14.5 \%$, a acompañar a algún adulto; $12.6 \%$, a limpiar; $10.3 \%$, a actuar; $5.4 \%$, a cuidar carros, y $11 \%$, a otras actividades, entre las que se encontraron lustrar zapatos, abrir puertas de los carros, llevar paquetes, prostituirse, dirigir tráfico vehi- 
cular y ayudar a peatones a pasar las calles, entre otras. Los niños más pequeños tuvieron mayor probabilidad de encontrarse acompañando a algún adulto o pidiendo limosna, mientras que el grupo de los más grandes estuvo compuesto básicamente por vendedores $(p=0.000)$. Las actividades que más se asociaron con el retraso escolar fueron, en orden descendente, vender, limpiar y pedir limosna. Las actividades que más se asociaron con falta de vinculación al sistema educati- vo fueron, en orden descendente, vender, limpiar y pedir limosna $(p=0.000)$.

\section{Características de género}

Los datos mostraron que las niñas entrevistadas tenían menor edad que los niños, estaban con mayor frecuencia acompañadas, permanecían menos tiempo durante la noche en la calle y estudiaban más que los

\section{Cuadro I}

Comportamiento de variables sociodemográficas en las ciudades capitales. Bogotá, ciudad
de México, Quito, San Salvador y ciudad de Guatemala (Noviembre, 2003-junio, 2004)

Ciudad Bogotá Ciudad de Guatemala_ Ciudad de México Quito San Salvador Total

$\begin{array}{lllllll}\text { Número de encuestas } & 478 & 120 & 97 & 177 & 100 & 972\end{array}$

Vivienda en calle (\%)

\begin{tabular}{cccccccc} 
No & 89.1 & 90.8 & 50.5 & 87 & 75 & 82.3 \\
\hline Sí & 10.9 & 9.2 & 49.5 & 13 & 25 & 17.7
\end{tabular}

Acompañante adulto $(\%)$

\begin{tabular}{lllllll} 
Solo & 44.8 & 35 & 66 & 42.4 & 59 & 46.7 \\
\hline Acompañado & 55.2 & 65 & 34 & 57.6 & 41 & 53.29
\end{tabular}

Seguridad social (\%)

\begin{tabular}{lcccccc} 
No asegurado & 63.8 & 99.2 & 91.8 & 50.3 & 100 & 72.2 \\
\hline Asegurado & 33.7 & 0 & 3.1 & 11.3 & 0 & 19 \\
\hline No sabe & 2.5 & 0.83 & 5.1 & 38.4 & 0 & 8.9
\end{tabular}

\section{Cuadro II}

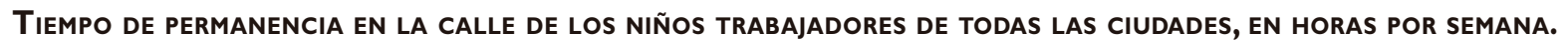
Bogotá, ciudad de México, Quito, San Salvador y ciudad de Guatemala (noviembre, 2003-junio, 2004)

\begin{tabular}{|c|c|c|c|c|c|c|c|c|c|c|}
\hline \multirow{3}{*}{$\begin{array}{l}\text { Total de niños con datos válidos } \\
\text { Horas en la calle por semana }\end{array}$} & \multicolumn{2}{|c|}{ Bogotá } & \multicolumn{2}{|c|}{ Ciudad de México } & \multicolumn{2}{|c|}{ Ciudad de Guatemala } & \multicolumn{2}{|c|}{ San Salvador } & \multicolumn{2}{|c|}{ Quito } \\
\hline & \multicolumn{2}{|c|}{478} & \multicolumn{2}{|c|}{97} & \multicolumn{2}{|c|}{120} & \multicolumn{2}{|c|}{100} & \multicolumn{2}{|c|}{177} \\
\hline & Numero & $\%$ & Número & $\%$ & Número* & $\%$ & Número & $\%$ & Número & $\%$ \\
\hline Menos de 10 horas & 12 & 2.5 & 0 & 0 & 0 & 0 & I & I & 4 & 2.26 \\
\hline De $10<20$ horas & 68 & 14.2 & I & I & 0 & 0 & I & I & 7 & 3.95 \\
\hline De $20<40$ horas & 119 & 24.9 & 6 & 6.2 & 27 & 22.7 & 43 & 43 & 79 & 44.6 \\
\hline 40 horas o más & 279 & 58.4 & 90 & 92.8 & 92 & 77.3 & 55 & 55 & 87 & 49.1 \\
\hline Trabajo nocturno menos de 10 horas & 3 & 0.6 & 7 & 7.2 & 3 & 25 & 0 & 0 & 14 & 8 \\
\hline Trabajo nocturno 10 o más horas & 100 & 21 & 70 & 72 & 0 & 0 & 13 & 13 & 27 & 15.3 \\
\hline
\end{tabular}

* Uno de los niños entrevistados en la ciudad de Guatemala no presentaba dato de horas trabajadas 


\section{Cuadro III}

Desplazamiento y desplazamiento por violencia en niños trabajadores de todas las ciudades. Bogotá, Ciudad de México, Quito, San Salvador y Ciudad de Guatemala (noviembre, 2003-junio, 2004)

\begin{tabular}{lccccc} 
& Bogotá & Ciudad de México & Ciudad de Guatemala & San Salvador & Quito \\
Total desplazados & 207 & 25 & 48 & 52 & 47 \\
\hline Desplazados por violencia & 192 & 13 & 9 & 43 & 0 \\
\hline Total niños entrevistados & 478 & 97 & 120 & 100 & 177 \\
\hline$\%$ niños entrevistados desplazados & 43.3 & 25.7 & 40 & 52 & 26.5 \\
\hline \% niños desplazados por violencia & 92.7 & 52 & 18.7 & 82.7 & 0
\end{tabular}

\section{Cuadro IV}

\section{Edad y género de la población encuestada. Bogotá, Ciudad de México, Quito, San Salvador} y Ciudad de Guatemala (noviembre, 2003-junio, 2004)

\begin{tabular}{|c|c|c|c|c|c|c|}
\hline Ciudad & Bogotá & Ciudad de Guatemala & Ciudad de México & Quito & San Salvador & Total \\
\hline \multicolumn{7}{|l|}{ Género (\%) } \\
\hline Masculino & 57.3 & 65 & 92.8 & 67.2 & 54 & 63.3 \\
\hline Femenino & 42.7 & 35 & 7.2 & 32.8 & 46 & 36.7 \\
\hline \multicolumn{7}{|l|}{ Edad (\%) } \\
\hline$<$ I año & 4.6 & 0 & 0 & 0 & 0 & 3.8 \\
\hline I-5 años & 27.4 & 4.2 & 3.1 & 5.1 & 9 & 14.6 \\
\hline 6-14 años & 59.9 & 88.3 & 63.9 & 84.7 & 79 & 64.2 \\
\hline $15-17$ años & 8.2 & 7.5 & 33 & 10.2 & 12 & 17.4 \\
\hline
\end{tabular}

hombres. Todas estas diferencias fueron estadísticamente significativas $(p=0.000)$ (cuadro IV).

\section{Características de edad}

La actividad realizada por los niños varió de acuerdo con la edad. Los infantes pequeños, por lo general, pedían limosna o acompañaban a un adulto. Los niños menores de un año y los mayores de 15 estaban presentes en la calle durante un lapso mayor. Los hijos de familias desplazadas eran de menor edad y eran aún más pequeños si la familia llevaba en la ciudad menos de un año. Todas estas diferencias fueron estadísticamente significativas $(p=0.000)$.

\section{Características de educación}

De los niños mayores de seis años, $47.4 \%$ no se encontraba estudiando en el momento de la encuesta. De los niños entre 6 y 14 años, 45.4\%, y 93.5\% de niños entre 15 y 17 años tenía un retraso superior a dos años escolares de acuerdo con lo esperado para su edad. A medida que aumentaba el número de horas que trabajaba el niño, menor fue su probabilidad de que estuviera estudiando en el momento de la encuesta $(p=0.000)$ (cuadro V).

\section{Análisis por ciudades}

En el cuadro I puede observarse el comportamiento de algunas variables sociodemográficas de las ciudades estudiadas.

Se encontraron más niños sin acompañante en la Ciudad de México y San Salvador. En la primera, la mayoría era del sexo masculino, mientras que en Bogotá y San Salvador hubo una diferencia ligera entre ambos sexos. En cuanto a la edad, la población infantil encontrada en Bogotá fue más joven y tenía mayor seguridad social. En relación con la vivienda, la mayoría vivía en la calle en la Ciudad de México y San Salvador. Todas estas diferencias fueron estadísticamente significativas $(p=0.000)$. Las actividades realizadas por 


\section{Cuadro $v$ \\ Estudio en NIÑos tRabajadores ENTRE 6 Y 17 aÑos de todas LAS CIUDAdes.* Bogotá, Ciudad de México, Quito, San Salvador y Ciudad de Guatemala (noviembre, 2003-junio, 2004)}

\begin{tabular}{|c|c|c|c|c|c|c|c|c|c|c|}
\hline & Bog & & Ciudad d & Téxico & Ciudad de & itemala & San Sal & & Qui & \\
\hline & Número & $\%$ & Número & $\%$ & Número & $\%$ & Número & $\%$ & Número & $\%$ \\
\hline Estudia & 282 & 59 & 4 & 4.4 & 31 & 25.8 & 44 & 44 & 115 & 65 \\
\hline No estudia & 143 & 30 & 90 & 92.7 & 84 & 70 & 47 & 47 & 53 & 30 \\
\hline Total & 425 & 100 & 94 & 100 & 115 & 100 & 91 & 100 & 168 & 100 \\
\hline
\end{tabular}

los niños en las ciudades capitales pueden verse en la figura 1.

El desplazamiento y el desplazamiento por violencia se comportaron de manera diferente. En la figura 2 pueden observarse las diferencias. Los niños entrevistados en Quito no reportaron desplazamiento por violencia en ningún caso, mientras que casi todos los de Bogotá y San Salvador lo hicieron $(p=0.000)$.

En la figura 3 puede observarse la afiliación al sistema educativo de los niños mayores de seis años en cada una de las ciudades capitales. Las diferencias son significativas entre las ciudades en cuanto a pertenencia o no al sistema educativo y retraso escolar. La que presenta mayor retraso escolar es la ciudad de México (87.2\%), seguida por San Salvador (79.1\%) y Ciudad de Guatemala (56.5). Estas diferencias fueron significativas $(p=0.000)$.

Se determinó una prevalencia de jornadas laborales extensas, mayores o iguales a medio tiempo, de $100 \%$ para Guatemala; $99 \%$ para la Ciudad de México; 98\% para San Salvador; $93.8 \%$ para Quito, y $83.3 \%$ para Bogotá.

En el análisis de correspondencias (figura 4), se observa cómo se agrupa el desplazamiento con niños menores de cinco años y acompañamiento por parte de adultos, características especialmente cercanas a Bogotá. Vivienda en la calle, falta de estudio, niños entre 15 y 17 años y retraso escolar se agrupan y están cercanas a la Ciudad de México. Estudio, no retraso escolar, falta de seguridad social, niños entre 6 y 14 años se agrupan y están cercanos a Quito. Alrededor de San Salvador se agrupan el retraso escolar y las niñas; finalmente, Guatemala se encuentra cercana a niños solos, edad de 6 a 14 y desplazamiento.

\section{Discusión}

El estudio presenta dos limitaciones importantes. a) La población de menores que trabajan en las calles es flo-

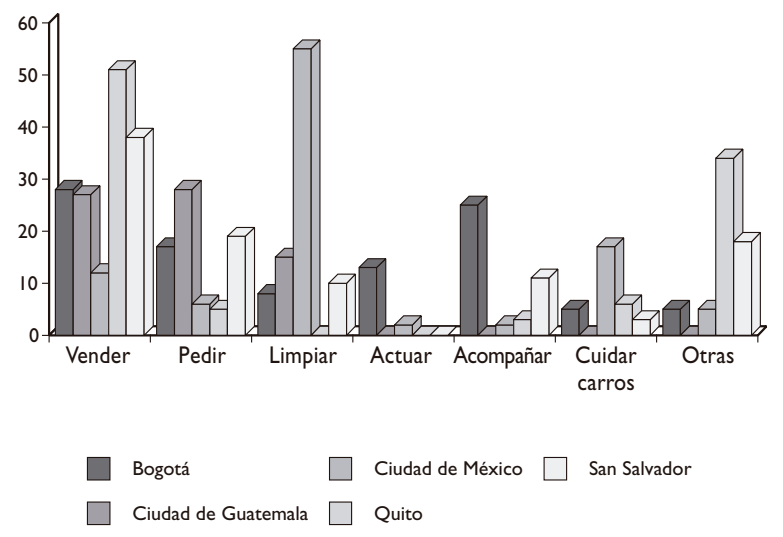

Figura I. Porcentaje de Niños en CADA ACtIVIDAd POR ciudad capital. Bogotá, Ciudad de México, Quito, San Salvador y Ciudad de Guatemala (noviembre, 2003-JUNIO, 2004

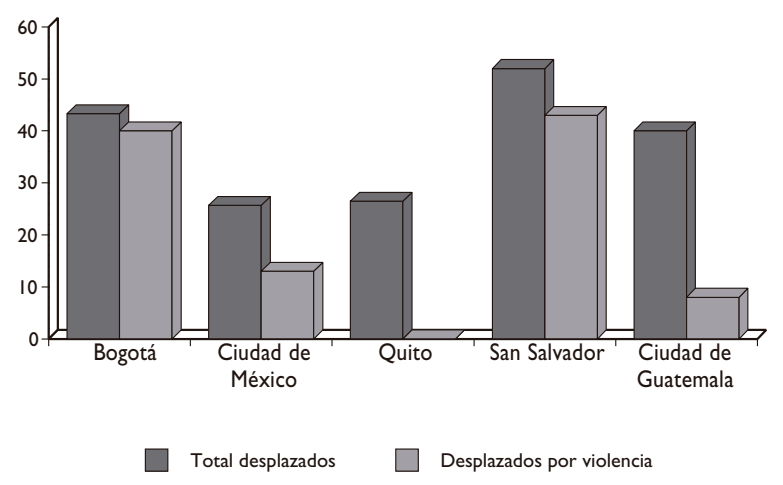

Figura 2. Fenómeno de desplazamiento. Total de DESPLAZADOS VS. DESPLAZADOS POR VIOLENCIA. BOGOTÁ, Ciudad de México, Quito, San Salvador y Ciudad de Guatemala (Noviembre, 2003-junio, 2004)

salud pública de méxico / vol.48, no.5, septiembre-octubre de 2006 


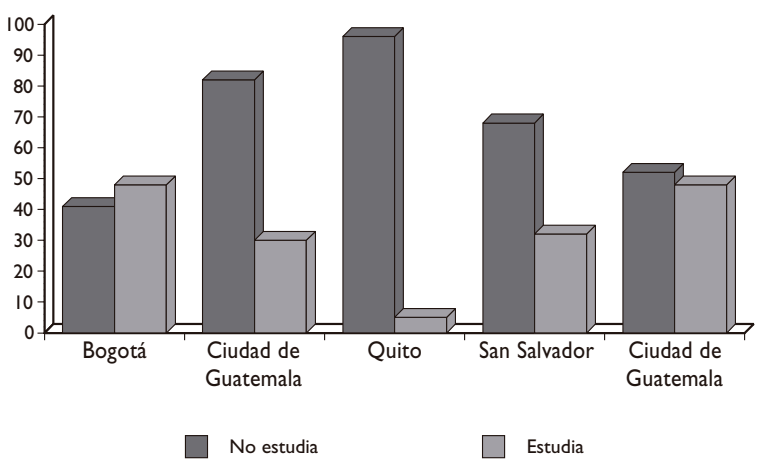

Figura 3. Pertenencia al sistema educativo por ciudad capital. Bogotá, Ciudad de México, Quito, San Salvador y Ciudad de Guatemala (NOViembre, 2003JUNIO, 2004)

tante y no siempre se encuentran los mismos niños, lo cual dificulta la selección de menores a estudiar y entorpece la generalización de los resultados. b) La selección de las calles para entrevistar a los niños fue difícil; a pesar de conseguir un consenso de las calles seleccionadas en cada ciudad entre quienes participaron en el estudio, esta selección puede contribuir a las diferencias encontradas. Sin embargo la información obtenida durante el mismo permite acercarse al conocimiento del fenómeno.

Otra limitación consiste en el hecho de haber recolectado la información durante los fines de semana. Aunque no existen estudios que lo sustenten de manera precisa, se sospecha que los fines de semana el número de niños es diferente al que se encuentra entre semana, y es posible que, incluso, se trate de dos poblaciones distintas; en este caso, sería necesario considerar diferencias en las variables escolaridad, compañía de la familia, edad, jornada laboral y actividad desarrollada.

Aproximadamente la mitad de los niños entrevistados no estaba acompañada por adultos (46.7\%). Esto, sumado al hecho de que en $66.9 \%$ de los casos los niños fueron quienes respondieron el cuestionario, nos muestra la gran independencia que tienen estos menores. El hecho de que las niñas se encontraran más acompañadas está relacionado con los riesgos que se presentan en las calles y las ideas de género que se manejan en nuestras culturas. ${ }^{18}$

De la población estudiada, $63.3 \%$ fue del sexo masculino, lo cual coincide con lo encontrado en otras

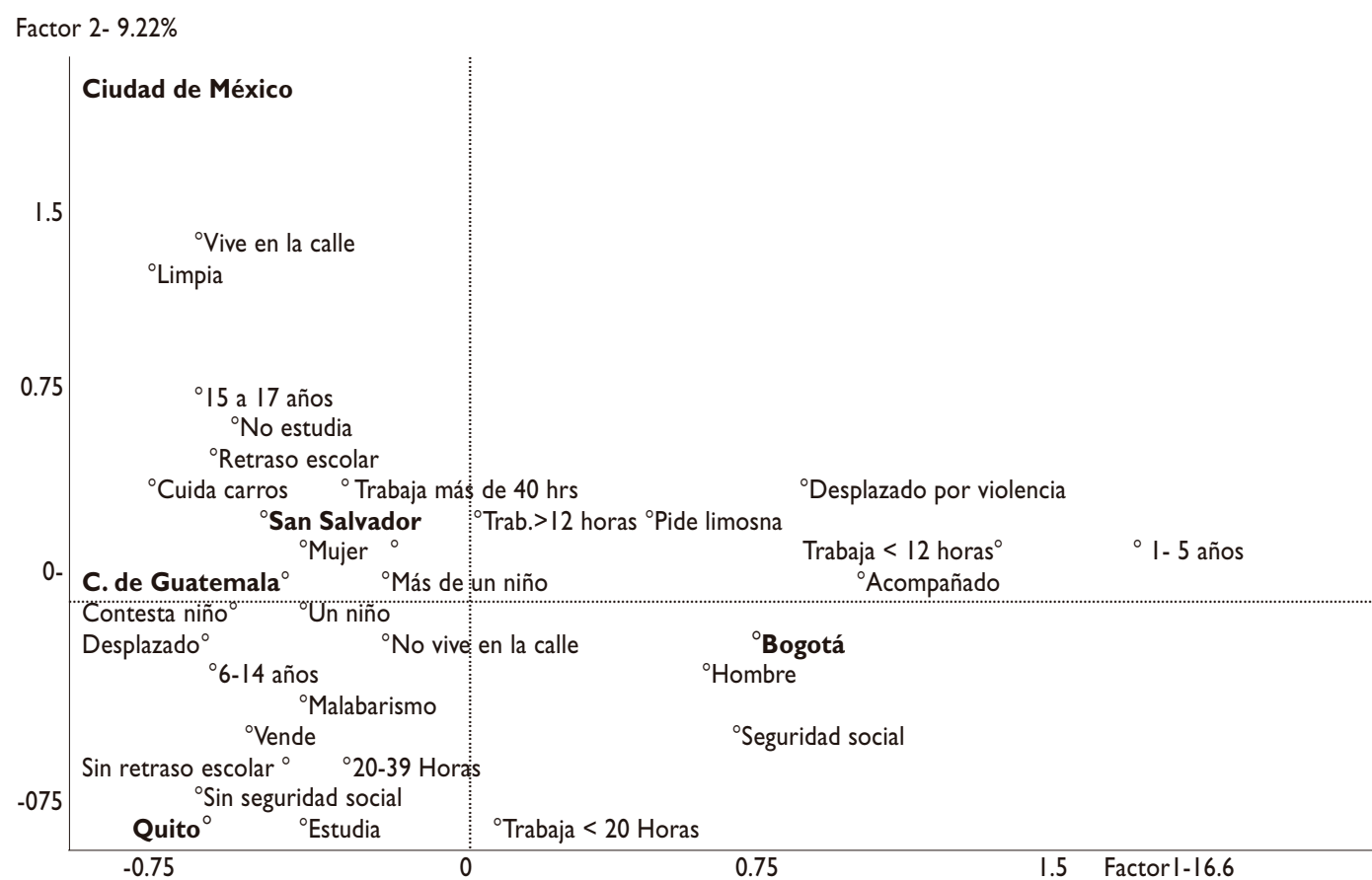

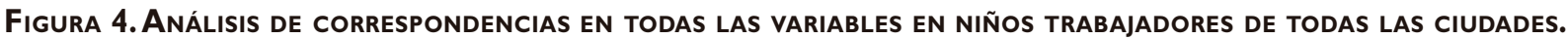
Bogotá, Ciudad de México, Quito, San Salvador y Ciudad de Guatemala (noviembre, 2003-junio, 2004) 
investigaciones. ${ }^{2,4,19,20}$ La mayoría de menores entrevistados perteneció al grupo de edad entre 6 y 14 años (64.2\%). Esto es importante ya que, de acuerdo con el Convenio 138 de la OIT, ${ }^{21}$ los niños menores de 15 años no deberían trabajar. Aunque la mayor parte de las caracterizaciones de estas poblaciones no incluye a menores de cinco años, este estudio sí lo hace debido a que se encuentran en condiciones similares a los mayores, y esto es de interés para la salud pública; además, el hecho de encontrarse en un ambiente similar al de sus hermanos o pares en edad pone en evidencia la necesidad de hacer una aproximación a sus condiciones de vida para entender el fenómeno y buscar mecanismos para erradicarlo. Los resultados de este estudio muestran el grado de desprotección en que se encuentran los niños, ya que muchos no tenían o desconocían si tenían seguridad social $(81 \%)$, trabajaban más de tiempo completo (62.1\%), lo cual coincide con lo reportado en la bibliografía, ${ }^{19}$ y trabajaban incluso durante la noche $(29.3 \%)$.

Los niños que provenían de familias desplazadas estaban en peores condiciones que los demás, ya que tenían menor seguridad social y estaban expuestos a los riesgos de la calle desde más corta edad. Este estudio muestra que el desplazamiento es un factor altamente relacionado con el trabajo infantil en la calle.

En relación con las actividades realizadas, vender fue la que desempeñaron con mayor frecuencia, lo cual es acorde con lo reportado en la bibliografía. ${ }^{19,22}$ El hecho de que los niños pequeños acompañen a un adulto o pidan limosna, mientras que los más grandes vendan, se relaciona con las habilidades adquiridas por los niños durante su desarrollo.

En este estudio no se tuvo en cuenta el nivel de ingresos; sin embargo, esta variable debería considerarse en estudios posteriores y relacionarse con la actividad laboral, la incidencia de esta actividad sobre el estudio y el retraso escolar, así como con el hecho de si el niño recibe el ingreso directamente o su acompañante.

El trabajo en la calle se asoció con baja escolaridad, lo cual coincide con otros estudios. ${ }^{3,20,23}$ A mayor número de horas de trabajo menor probabilidad de encontrarse estudiando.

Podemos concluir que cada una de las ciudades capitales tiene características especiales.

En Bogotá se encontraron los niños más pequeños y el desplazamiento por violencia fue muy importante, lo cual puede explicarse por el conflicto interno que vive el país. La mayoría de los niños no eran habitantes de la calle y la proporción de los que trabajaban menos de medio tiempo fue mayor. En la Ciudad de México, por el contrario, casi 50\% de los niños entrevistados era habitante de calle; $66 \%$ no estaba acom- pañado de un adulto; $99 \%$ laboraba más de medio tiempo, y $91.8 \%$ no tenía seguridad social. Esto deja ver que el problema del trabajo infantil en esta capital es grande, como lo ha reportado la UNICEF. ${ }^{7,8}$

En Ciudad de Guatemala, 35\% de los niños no estaba acompañado por un adulto en el momento de la entrevista; $40 \%$ era desplazado, y $8.3 \%$ era desplazado por la violencia; la totalidad trabajaba, por lo menos, medio tiempo; $56.5 \%$ tenía retraso escolar mayor a dos años, y 99\% carecía de seguridad social, datos que complementan las cifras anteriormente publicadas para Guatemala, donde aproximadamente $23 \%$ de los niños trabaja. ${ }^{24}$

En Quito predominó el desplazamiento por causas diferentes a la violencia, lo cual es coherente con la situación de país. Los niños se encontraron vendiendo y realizando otras actividades, lo cual confirma lo reportado por ellos acerca del alto porcentaje de niños que son vendedores ambulantes y lustrabotas. ${ }^{22}$ Esta ultima actividad, que no fue predominante en las otras ciudades, sí se manifestó en Quito y aumentó el porcentaje que se refiere a otras actividades, como puede verse en la figura 1.

En El Salvador, ningún niño tenía seguridad social y fue evidente el alto desplazamiento por violencia. Los datos sociodemográficos coincidieron con los reportados por estudios previos..$^{19}$ Esto, en lo que se refiere a edad y sexo de los entrevistados, da cuenta de la gran cantidad de niños trabajadores que están en la etapa de inicio de su actividad escolar, con todo lo que implica en su desarrollo, sobre todo cuando la jornada laboral es larga y les impide su inserción en el sistema educativo.

El desplazamiento es un fenómeno de difícil análisis, debido a que se produce por causas diversas. Esto influye en el interés que despierta su estudio. Respecto a los desplazados por violencia, las cifras más alarmantes presentadas por el Norwegian Refugee Council corresponden a Colombia, Perú y México durante 2004. Es así como en Colombia se calcula un número de 290000 desplazados para ese año; 60000 para Perú, y 10000 a 12000 para México. En Colombia la situación es especialmente grave, ya que es el tercer país del mundo en desplazamiento interno acumulado. ${ }^{25}$ Llama la atención que no se encuentren estadísticas actuales de desplazamiento interno en El Salvador; ${ }^{26}$ las referencias encontradas datan de los años 90 y consideran el desplazamiento interno en este país como un problema superado; sin embargo, nuestro estudio sugiere una situación totalmente opuesta al encontrar un alto porcentaje de niños desplazados por la violencia. Este es un aspecto a profundizar que deberá analizarse con más detalle con datos históricos. Otra 
explicación a considerar sería que el acompañante del niño que contestó la entrevista provenga de una familia desplazada por la violencia en los años de la guerra, o que en los análisis de la ACNUR de las Naciones Unidas no se consideren estadísticas de desplazados por violencia de carácter diferente a la producida por la guerra.

El desplazamiento forzado por violencia o el generado por miseria es un fenómeno que a todas luces debe estudiarse como factor causal explicativo del trabajo infantil, ya que entre 25 y 50\% de los niños entrevistados refirieron esta condición, como puede observarse en la figura 2. Asimismo, en las formas de prevención y contención de este fenómeno, debe encontrarse gran parte de la solución al flagelo del trabajo infantil; por lo tanto, esta es una línea de estudio e intervención que debe considerarse para futuros análisis.

Las ciudades donde se encontró una mayor prevalencia de jornadas laborales extensas son la Ciudad de México y Bogotá, lo cual nos permite concluir que en estas ciudades los menores presentan una mayor exposición a factores de riesgo.

El ámbito laboral de nuestros entrevistados es el espacio público, por lo tanto, no sólo no tienen ningún tipo de seguridad, sino que tienen que evadir peligros; tienen que disputarse su sitio de trabajo con el resto de la población y muchas veces tienen que pagar por el mismo; ${ }^{2}$ constantemente están expuestos a ser arrollados y al abuso sexual. Este sitio de trabajo genera, además, exposición a situaciones violentas derivadas de la inseguridad social, la persecución por parte de las autoridades y el encuentro con indigentes habitantes de la calle y delincuentes. ${ }^{27}$ Diversos estudios han encontrado que los niños de la calle son con frecuencia forzados a prostituirse, ${ }^{28,29}$ lo que sugiere que una de las consecuencias desfavorables del trabajo infantil en la calle, incluso en la modalidad de ingreso familiar suplementario, es el incremento de la exposición al riesgo de abuso o explotación sexual, que puede llegar hasta la trata de niños. Además de la falta de seguridad, estos niños están expuestos a otros factores de riesgo entre los que se encuentran los físicos (ruido, temperaturas extremas, radiaciones), químicos (humos, ${ }^{30,31}$ material particulado ${ }^{2}$ ), ergonómicos (sobreesfuerzos, movimientos forzados, trabajo prolongado de pie, vocear) y psicosociales (jornada laboral extensa, ansiedad, agresión, estrés, acoso sexual y fatiga). 2,3,32,33

La conclusión principal de esta investigación es que el fenómeno de trabajo infantil ambulante en las capitales latinoamericanas no es unívoco. Dicho fenómeno se comporta de manera diferente en cada ciudad. La mayoría de los cruces estadísticos por ciudades mostraron diferencias significativas. Como se trabajaron muestras de tamaño distinto para cada ciudad, las comparaciones que pretendan realizarse entre ellas deben hacerse con precaución; lo más adecuado es comparar cada una con el promedio de las ciudades. De cualquier manera, para un análisis más preciso se presentan datos proporcionales del comportamiento de las variables respecto al número de niños entrevistados por ciudad.

El principal corolario que parece desprenderse de esta conclusión es que las medidas para enfrentar el fenómeno del trabajo infantil ambulante deben desarrollarse caso por caso. Si bien en esta investigación no se estudiaron las causas del fenómeno, los resultados del estudio sugieren la pluralidad y especificidad de factores. En consecuencia, los resultados sugieren que las soluciones deben tener un alto contenido de especialidad, de suerte que respondan adecuadamente a las particularidades del fenómeno en cada ciudad.

No obstante lo anterior, existen elementos comunes que ocurren en los diferentes países. El primero de ellos es la presencia del fenómeno en todas las ciudades estudiadas. El segundo es la existencia de una situación general muy desfavorable para los niños trabajadores ambulantes. Si bien hay variaciones significativas en el comportamiento de las variables, en las ciudades estudiadas los niños están expuestos a condiciones peligrosas e inadecuadas, incluyendo jornadas excesivas, bajas tasas de escolaridad, y baja cobertura del sistema de seguridad social.

Al comparar el comportamiento de ciertas variables por ciudad con la media encontramos situaciones que es necesario considerar en relación con las políticas sociales y sanitarias públicas. En el caso de la ciudad de México, por ejemplo, el alto porcentaje de niños que manifestó vivir en la calle debe analizarse, ya que existen estudios que revelan el alto riesgo de los niños que están en condiciones de indigencia, ya que el desprendimiento de la familia implica un mayor riesgo de consumo de sustancias psicotrópicas y desarrollo de actividades ilegales. ${ }^{34}$

Con base en lo anterior puede concluirse que el trabajo infantil ambulante en las capitales latinoamericanas estudiadas puede considerarse una actividad peligrosa, que se enmarca dentro de las peores formas de trabajo infantil de acuerdo con los estándares internacionales. ${ }^{35}$

\section{Agradecimientos}

Agradecemos a Franklin Libenson, Pedro José y Ana María Moran, Alfredo Larrea y Mónica Rojas, Catalina Latorre, María Clara Rondón, Carlos Enrique Rivera y Camilo Moreno por su colaboración. 


\section{Referencias}

I. Organización Internacional del Trabajo. La acción del IPEC contra el trabajo infantil: Hechos sobresalientes. Ginebra: OIT, 2002.

2. UNICEF Colombia, Universidad Nacional de Colombia. Creciendo en el asfalto. Niños, niñas y jóvenes vendedores en las calles de Bogotá. Bogotá: Universidad Nacional de Colombia, 2002.

3. Power C, Stansfeld SA, Matthews S, Manor O, Hope S. Childhood and adulthood risk factors for socio-economic differentials in psychological distress: evidence from the 1958 British birth cohort. Soc Sci Med 2002; 55(II):1989-2004.

4. Delgado MC. La realidad del trabajo infantil en las calles de la ciudad de Neiva, desde una perspectiva etnográfica. Neiva: Universidad Surcolombiana, Asesográficas, 2003.

5. Departamento Administrativo Nacional de Estadística, Programa Internacional para la Erradicación del Trabajo Infantil Sudamérica. Encuesta Nacional de Trabajo Infantil. Bogotá: DANE-OIT, 200I.

6. García E. Derecho de la infancia-adolescencia en América Latina. De la situación irregular a la protección integral. Bogota: UNICEF, 1997.

7. Fondo de las Naciones Unidas para la Infancia. El trabajo infantil en México. UNICEF. Disponible en: http://www.unicef.org/mexico/ programas/trabajo/htm.

8. Casa Alianza. Trabajo infantil: visión de conjunto. Bogota: Casa Alianza, 2002. Disponible en: http://casa-alianza.org/ES/human-rights/laborexploit/.

9. Organización Internacional del Trabajo. La Acción del IPEC contra el trabajo infantil. Ginebra: OIT, 2004-2005. Disponible en: http://

www.ilo.org/iloroot/docstore/ipec/prod/spa/

200602_implementationreport_es.pdf.

10. Parker DL. Street children and child labour around the world. Lancet. 2002, dic 2I-28; 360(9350):2067-207I.

II. Organización Internacional del Trabajo. Understanding children's work in El Salvador. Ginebra: OIT, 2004. Disponible en: http:// www.ilo.org/public/english/standards/ipec/simpoc/elsalvador/document/ esnationalreporten2004.pdf.

12. Pinzón AM, Briceño L, Gómez Al, Latorre C. Trabajo infantil en las calles de Bogotá. Rev Cienc Salud 2003; I (2):5I-63.

13. Programa Internacional para la Erradicación del Trabajo Infantil Organización Internacional del Trabajo. Trabajo infantil urbano en El Salvador: una evaluación rápida. Ginebra: OIT, 2002. Disponible en: http://www.oit.org.pe/ipec/documentos/elsalv_ti_urb.pdf.

14. Programa Internacional para la Erradicación del Trabajo Infantil Organización Internacional del Trabajo. Trabajo infantil en la floricultura en Ecuador: una evaluación rápida. Ginebra: OIT, 2002. Disponible en: http://www.oit.org.pe/ipec/documentos/floricult_ecuador_vf.pdf. 15. Programa Internacional para la Erradicación del Trabajo Infantil Organización Internacional del Trabajo. Trabajo infantil en los basureros en Guatemala: una evaluación rápida. Ginebra: OIT, 2002. Disponible en: http://www.oit.org.pe/ipec/documentos/gu_basuras_ras.pdf. 16. Fondo de las Naciones Unidas para la Infancia, Programa de las Naciones Unidas para la Fiscalización Internacional de Drogas, Desarrollo Integral de la Familia. Estudio de niñas, niños y trabajadores en 100 ciudades. México: UNICEF, 1999. Disponible en: http:// www.unicef.org/mexico/programas/censoninostrabajadores $100 \mathrm{cdes}$.pdf. 17. Programa Internacional para la Erradicación del Trabajo InfantilOrganización Internacional del Trabajo. Estadísticas por país. Lima, San José de Costa Rica: OIT, 2006. Disponible en: http://www.oit.org.pe/ipec/ pagina. php?seccion $=44 \&$ pagina $=175$.

18. Stark E, Flitcraft AH. Women and children at risk: a feminist perspective on child abuse. Int J Health Serv. 1988; I8(I):97-II8. 19. Quintero H, Rivas W. El Salvador: trabajo infantil urbano: una evaluación rápida. Ginebra: OIT, 2002.

20. Aderinto AA. Social correlates and coping measures of street children: a comparative study of street and non-street children in South-Western Nigeria. Child Abuse Negl 2000;24(9):I I99-12I3.

21. Organización Internacional del Trabajo. Convenio sobre la edad mínima, No. 138. (6 de junio de 1973).

22. Venegas de la Torre F. Los conceptos, estrategias y metodologías en relación al trabajo infantil. Quito: Instituto Nacional de la Niñez y la Familia, 2002. Disponible en: http://www.monografias.com/trabajos /2/ trabinf/trabinf.shtml.

23. Laniano-Laborin R, Moreno C,Vargas R, Deosaransingh K, Woodruff $\mathrm{SI}$, Sallis JF. Los niños que trabajan en las calles de Tijuana. Perfil epidemiológico y prevalencia de experimentación con tabaco. Salud Publica Mex 1995;37(2): 149-I54.

24. Oficina Internacional de Trabajo. Encuesta del trabajo infantil en Guatemala. Ciudad de Guatemala: OIT, 200I.

25. Eschembacher JH. Internal displacement. Global overview of trend developments in 2004. Ginebra: Global IDP Project. Norwegian Refugee Council, 2005.

26. Internal Displacement Monitory Center. Global statistics. Norwegian Refugee Council; 2005. Disponible en: http://www.internal-

displacement.org/8025708F004CE90B/(httpPages)/ 22FBID4E2B I96DAA802570BB005E787C? OpenDocument\&count $=1000$. 27. Pick WM, Ross MH, Dada Y.The reproductive and occupational health of women street vendors in Johannesburg, South Africa. Soc Sci Med 2002 enero;54(2):193-204.

28. Irwanto S. Street children in Indonesia. Lancet 2002;360:I508. 29. Organización Internacional del Trabajo. Entendiendo el trabajo Infantil en Guatemala. Ciudad de Guatemala: OIT, 2003.

30. Szaniszlo J, Ungvary G. Polycyclic aromatic hydrocarbon exposure and burden of outdoor workers in Budapest. J Toxicol Environ Health A 200I;62(5):297-306.

31. Duarte-Davidson R, Courage C, Rushton L, Levy L. Benzene in the environment: an assessment of the potential risks to the health of the population. Occup Environ Med 2001;58(I):2-13.

32. Salazar MC. Niños y jóvenes trabajadores buscando un futuro mejor. Bogotá: Universidad Nacional de Colombia-Unicef, 1990.

33. Hernández-Peña P, Kageyama ML, Coria I, Hernández B, Harlow S. Condiciones de trabajo, fatiga laboral y bajo peso al nacer entre vendedores ambulantes. Salud Publica Mex 1999; 4I (2): 101-109. 34. Departamento Administrativo Nacional de Estadistica. Censo de menores trabajadores de la calle y en la calle. Medellín: DANE, 200I. 35. International Labor Organization. Statistics on working children and hazardous child labour in brief. Geneva: ILO, 1998. 